\title{
Anemia carencial y SIDA
}

\author{
OSCAR RUIZ ${ }^{1}$, DAVID DÍAZ ${ }^{1}$, OSCAR CASTILLO ${ }^{1}$, RAFAEL REYES ${ }^{1}$, \\ MANUELA MARANGONI ${ }^{2}$, GERARDO RONCEROS ${ }^{1}$ \\ ${ }^{1}$ Instituto de Investigaciones Clínicas-UNMSM. ${ }^{2}$ Programa PROCETSS, Hospital Nacional Dos de Mayo.
}

\begin{abstract}
RESUMEN
OBJETIVOS: Determinar el tipo mas frecuente de anemia en pacientes con SIDA y el grado de severidad de la anemia. MATERIAL Y MÉTODOS: Se estudió 100 pacientes, entre 18 y 60 años, infectados por virus de inmunodeficiencia humana (VIH) en estadio SIDA, de Lima Metropolitana y el Callao, desde enero a diciembre 2001. Se realizó hemograma, mielograma, dosaje sérico de hierro, saturación de transferrina, ferritina, folato y vitamina B12. Las muestras fueron procesadas en el Departamento de Patología Clínica del Hospital Nacional Dos de Mayo. RESULTADOS: De los 100 pacientes, 60\% presentaron anemia severa, 30\% moderada y $10 \%$ leve. La tipificación del cuadro anémico fue carencial en $70 \%$ y por enfermedad crónica en $30 \%$. En el caso de anemia carencial, 25\% fue ferropénica, 30\% carencial mixta (ferropénica y megaloblástica) y $15 \%$ megaloblástica. De los casos con componente megaloblástico, 30 pacientes tuvieron deficiencia de folatos y 15 carencia de vitamina B12. CONCLUSIONES: La anemia prevalente fue la del tipo carencial. El grado de anemia predominante fue el severo.
\end{abstract}

Palabras clave: Anemia; síndrome de inmunodeficiencia adquirida; trastornos nutricionales.

\section{NUTRITIONAL ANEMIA AND AIDS SUMMARY}

OBJECTIVES: To determine the type of anemia most frequent in patients with AIDS and the various degrees of anemia. MATERIAL AND METHODS: One hundred patients 18 to 60 year-old infected with human immune deficiency virus (HIV) with residence in Lima and Callao were studied from January to December 2001 for blood count bone marrow aspiration, serum iron, transferrin, ferritin, folate and vitamin B12 levels. Samples were evaluated at the "Dos de Mayo" Hospital Clinical Pathology Department. RESULTS: From the 100 patients, 60\% had severe, $30 \%$ moderate and 10\% light anemia; $70 \%$ nutritional and $30 \%$ due to chronic disease. In the nutritional anemia group, $25 \%$ was ferropenic anemia, 30\% ferropenic and megaloblastic (double defect) and 15\% megaloblastic. In the megaloblastic anemia group, 30 patients had folate deficiency and 15 vitamin B12 deficiency. CONCLUSIONS: Prevalent anemia was nutritional. Anemia's degree was frequently severe.

Key words: Anemia; acquired inmunodeficiency syndrome; nutrition disorders.

\section{INTRODUCCIÓN}

Los virus están asociados desde hace mucho tiempo a desórdenes hematológicos. Reciente-

\footnotetext{
Correspondencia:

Dr. Oscar Ruiz Franco

Hospital Nacional Dos de Mayo

Instituto de Investiaciones Clínicas - UNMSM

Parque de la Medicina s/n. Lima 43, Perú

E-mail: osma@ec-red.com
}

mente los virus de los linfocitos " $\mathrm{T}$ " humanos (HTLV o Human T-lymphocyte virus) han sido asociados a varias afecciones hematológicas; así, el virus HTLV-1 a la leucemia linfocílitico crónica, el virus HTLV-2 a la leucemia a células peludas o tricoleucemia y el HTLV-3 (actualmente llamado HIV-1) al SIDA $\left({ }^{1-4}\right)$. La molécula CD4 es el principal receptor para el virus HIV-1, por lo tanto las células que la albergan, incluidos los linfocitos " $T$ ", los 
monocitos, los macrófagos tisulares, las células dendríticas y los megacariocitos, son aquellas prevalentemente infectadas por el virus. La célula progenitora CD34+ tiene un pequeño número de moléculas de superficie $\mathrm{CD} 4$, pero probablemente la infección viral no tiene significado clínico. Si bien no se ha probado aún de modo concluyente, se sabe que el virus induce apoptosis, destruyendo así la población de los linfocitos CD4+. Además la interacción del virus con la superficie celular de las células CD34+ puede inducir apoptosis y por lo tanto contribuye a la reducción del número de precursores celulares disponibles $\left(^{3-8}\right)$.

Las anomalías hematológicas presentes reflejan no sólo el efecto del virus sino también anormalidades de la modulación de la hemopoyesis. Se ha descrito anomalías de los modulares intracelulares, como disminución de la eritropoyetina y del factor estimulante de las colonias de los granulocitos-macrófagos y un aumento de la interleucina $6\left({ }^{9-13}\right)$. La infección del virus de inmunodeficiencia humana (VIH) puede inducir anemia, neutropenia, trombocitopeniao pancitopenia. Es importante tener presente que una anormalidad hematológica de un paciente con SIDA puede ser multifactorial y no sólo estar presente en relación a la infección $\left({ }^{3,5-7,14-16}\right)$.

La anemia en los pacientes con SIDA se constituye en la alteración hematológica más frecuente, llegando a tener una prevalencia que varía entre el $3,2 \%$ en los pacientes asintomáticos hasta $21 \%$ en sujetos con un recuento de las CD4 inferior a $249\left(^{3-5,14,17-20}\right)$. La literatura anglosajona, en el caso de anemia, describe como la más frecuente a aquella ligada a enfermedad crónica en las personas con SIDA. También se describe cuadros relacionados a anemia carencial (por deficiencia de hierro, folato y vitamina B12) ${ }^{21,22}$ ) y a anemia hemolítica del tipo inmune $\left(^{3-5,23}\right)$. En nuestro país, dadas las condiciones socioeconómicas en que vive la mayoría de la población, cobran una gran importancia las anemias carenciales en los pacientes con SIDA, pues una vez diagnosticadas y con un aporte del suplemento necesario, se mejoraría la calidad de vida de estos pacientes. Es importante estudiar el síndrome anémico en los enfermos con SIDA, pues en el Perú, como en la mayoría de los países en vías de desarrollo, la enfermedad sigue aumentando, a diferencia de los países desarrollados en donde el cuadro se ha estabilizado; por lo tanto, los especialistas debemos tratarcon mayor frecuencia a este tipo de pacientes.

La situación socioeconómica del Perú contribuye a que las anemias carenciales debidas a deficiencia de hierro, folato y vitamina B 12 cobren gran importancia en este estudio, desplazando a la anemia por enfermedad crónica descrita como la más frecuente en los países desarrollados para su población infectada con el virus del SIDA.

Los objetivos del presente trabajo son:

1) Determinar el tipo más frecuente de anemia en pacientes con SIDA.

2) Determinar el grado de severidad de la anemia encontrada en pacientes con SIDA.

\section{MATERIAL Y MÉTODOS}

El trabajo de investigación se realizó en pacientes infectados por por el virus de inmunodeficiencia humana (HIV) en estadio síndrome de inmunodeficiencia adquirida (SIDA) que acudieron para atención a los hospitales de Lima metropolitana y el Callao, durante el periodo comprendido entre enero y diciembre 2000.

El tipo de estudio fue descriptivo, prospectivo, de corte transversal.

La población estuvo conformada por 100 pacientes con infección VIH en estadio SIDA que cumplieron con los criterios de selección establecidos.

Los criterios de inclusión para el Diagnóstico de SIDA fueron: Prueba confirmatoria para HIV positiva: Wester Blot, recuento de CD4 menor de $200 \mathrm{cel} / \mathrm{mL}$, enfermedad indicatoria de SIDA. 
Para el diagnóstico de anemia, niveles de hemoglobina en relación a anemia, según la Organización Mundial de la Salud (OMS), es decir, hemoglobina $(\mathrm{Hb})$ menor de $14 \mathrm{~g} / \mathrm{dL}$ en varones adultos, $\mathrm{Hb}$ menor de $12 \mathrm{~g} / \mathrm{dLen}$ mujeres adultas, $\mathrm{Hb}$ menor de $11 \mathrm{~g} / \mathrm{dL}$ en gestantes y $\mathrm{Hb}$ menor de $12 \mathrm{~g} / \mathrm{dL}$ en niños menores 6 años. Los criterios de exclusión fueron: Pérdidas sanguíneas evidentes en los 3 meses previos al estudio, haber recibido transfusiones sanguíneas o tratamiento con suplementos de hierro, ácido fólico y vitamina B12 seis meses previos al estudio.

Se determinó el hemograma procesado con auto analizador Cell Dyn 1,700 de laboratorios Abbott, aspirado de médula oséa en caso de confirmar anemia, dosaje de hierro sérico, saturación de transferrina y ferritina sérica, dosaje de folato sérico y dosaje de vitamina B12.

Las muestras fueron procesadas en los diferentes ambientes del departamento de Patología Clínica del Hospital Nacional Dos de Mayo.

Según los resultados de la hemoglobina la anemia fue clasificada en leve (varones adultos 10 a $13,9 \mathrm{~g} / \mathrm{dL}$, mujeres adultas 10 a $11,9 \mathrm{~g} /$ $\mathrm{dL}$, gestantes 10 a $10,9 \mathrm{~g} / \mathrm{dL}$, niños menores de 6 años 10 a $11,9 \mathrm{~g} / \mathrm{dL}$ ), moderada ( 8 a 9,9 g/ dL) y severa (menor de $8 \mathrm{~g} / \mathrm{dL}$ ).

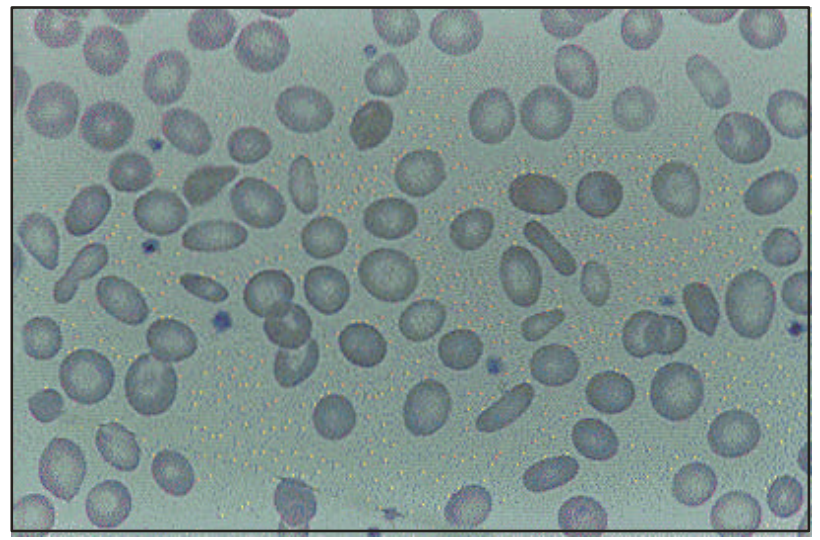

Figura 2.- Frotis periférico: microcitosis e hipocromía en anemia ferropénica.
Se realizó gráficos porcentuales circulares para expresar el porcentaje del grado de anemia encontrados en el estudio; así como para esquematizar la frecuencia de cada tipo de anemia.

\section{RESULTADOS}

Se estudió 100 pacientes en estadio SIDA. De ellos, 65 fueron varones y 35 mujeres. La edad de los sujetos testados fluctuó entre los 18 y 60 años. No se tuvo gestantes y niños dentro del estudio. De los 100 pacientes, 60 presentaron anemia severa (60\%), 30 anemia moderada $(30 \%)$ y 10 anemia leve (10\%) (Figura 1$)$.

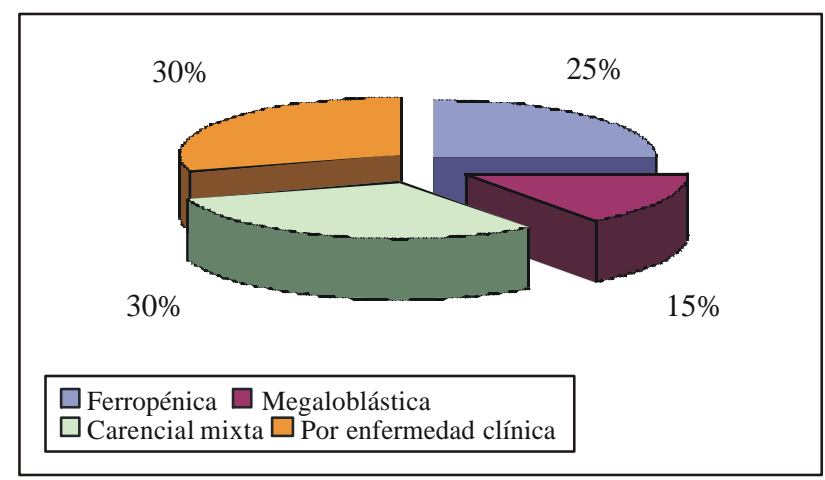

Figura 1.- Tipo de anemia en SIDA.

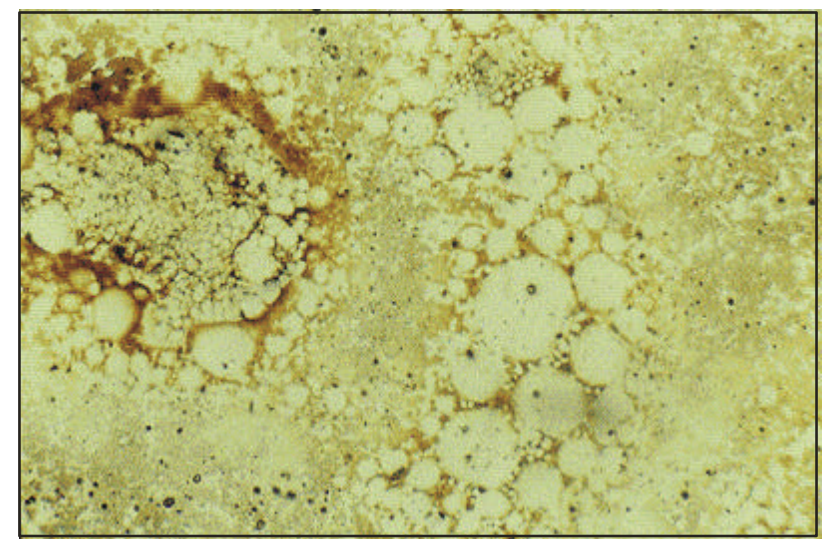

Figura 3.- Médula ósea: ausencia de los depósitos de hierro en anemia ferropénica. 
La tipificación del cuadro anémico fue: anemia carencial en 70 pacientes $(70 \%)$ y anemia por enfermedad crónica para 30 de ellos (30\%).

De las 70 personas con anemia carencial, 25 tuvieron anemia ferropénica (Figuras 2 y $3), 30$ presentaron anemia carencial mixta (anemia ferropénica y megaloblástica) y 15 cursaron con anemia megaloblástica (Figuras 4,5 y 6). De los 45 pacientes con componente megaloblástico, 30 tuvieron deficiencia de folatos y 15 de ellos carencia de vitamina B 12 . De los 15 pacientes con anemia megaloblástica pura, 4 tuvieron tanto deficiencia de folato como de vitamina B12.

Los 30 pacientes con anemia por enfermedad crónica tuvieron hierro sérico bajo y ferritina alta.

En este trabajo no se reporta casos de aplasia medular y de anemia hemolítica de tipo inmune.

\section{DISCUSIÓN}

El virus del SIDA afecta las células que contienen la molécula CD4, la que se encuentra en las células sanguíneas y hematopoyéticas; por lo tanto, al interactuar con algunas de ellas produce apoptosis, lo que sumado a las anormalidades en la regulación de la hematopoyesis puedenocasionar desde anemia hasta pancitopenia $\left(^{3-5}\right)$. La literatura internacional describe como anemia más frecuente la conocida como anemia por enfermedad crónica $\left({ }^{4,5,14,23-25}\right)$, la que se caracteriza por una mala utilización del hierro (hierro sérico disminuido y ferritina sérica alta). En este estudio, los hallazgos evidencian que la anemia que predomina en la población estudiada es la llamada carencial, en 70\%. Una explicación para ello sería que la mayoría de los pacientes con SIDA partenece a un nivel socioeconómico bajo, lo que influye en su estado nutricional y en las condiciones de salubridad en que viven. Otro factor seria que en el Perú la mayoría de pacientes con infección VIH, y más aun en estadio SIDA, no recibe tratamiento antirretroviral, hecho que los pone en desventaja en comparación con aquellos que sí lo reciben en los países desarrollados $\left({ }^{1}\right)$. Todos estos factores estarían a favor de una multicausalidad en la génesis de la anemia.

El protocolo del programa de atención integral del paciente con infección VIH/SIDA no contempla dentro de su manual las manifestaciones hematológicas de estos pacientes. La literatura internacional describe que la anemia es la manifestación hematológica más frecuente; por lo tanto, este trabajo pretende dar luces en relación a la etiología de la anemia en los pacientes con SIDA, haciendoénfasis en que el componente carencial, por las características socioeconómicas de la población, es un factor importante en su génesis.

El grado de anemia predominante fue el severo, con un $60 \%$ del total. Este aspecto podría dificultar el tratamiento de esta patología, en el sentido que muchas veces los pacientes requieren de hospitalización para recibir transfusión de paquetes de glóbulos rojos y luego dar el suplemento vitamínico carente.

El componente ferropénico se encuentra presente en $25 \%$ del total de pacientes estudiados. Las probables causas pueden ser el estrato socioeconómico deprimido al que pertenece la mayoría de los pacientes en estudio; por lo tanto, el acceso a los alimentos de origen animal así como a los servicios básicos y de salud es limitado.

El componente megaloblástico se presenta en 15 de ellos, siendo las posibles causas similares a lo mencionado para la carencia de hierro.

La carencia de folatos se evidencia en $10 \mathrm{de}$ los sujetos con anemia megaloblástica, pudiendo ser ocasionada por la frecuente presencia de diarrea crónica y el uso profiláctico del trimetropin/sulfametoxazol (quimioterápico que bloquea la acción de la enzima tetrahidrofolato reductasa), impidiendo la absorción de esta vitamina. 


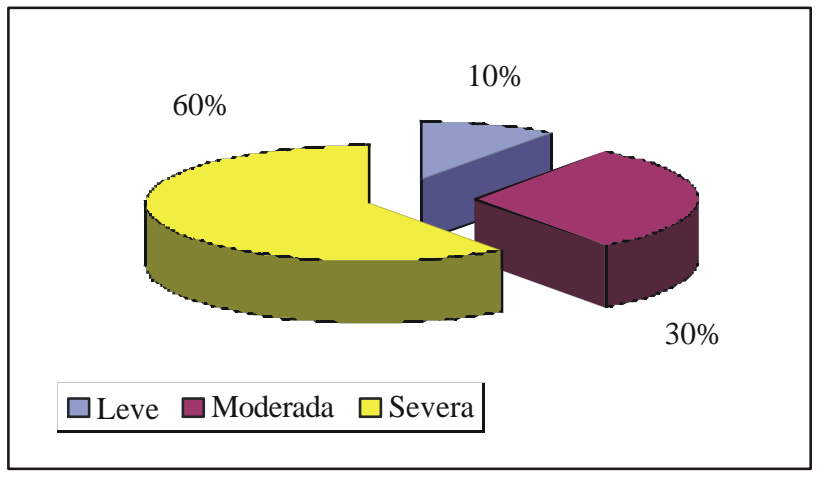

Figura 4.- Severidad de anemia en SIDA.

La deficiencia de vitamina B 12 se presentó en 5 pacientes, pudiendo ser las causas de su origen tanto las diarreas crónicas como el poco acceso de alimento de origen animal.

Podríamos sugerir las siguientes recomendaciones:

1) Tipificar el síndrome anémico en todos los pacientes infectados con VIH y sobre todo aquellos en estadio SIDA, para determinar un componente carencial.

2) Incentivar a través de charlas de educación sobre la importancia del régimen dietético,

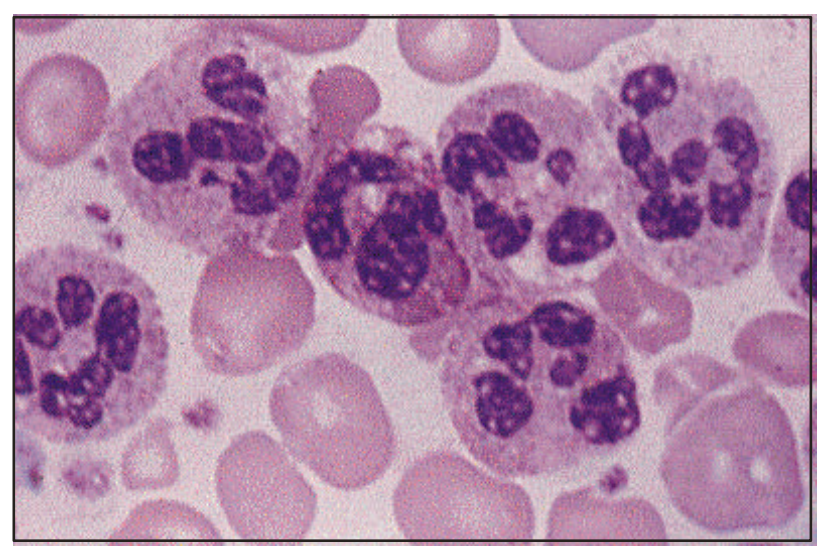

Figura 5.- Frotis periférico: macrocitosis e hipersegmentación de neutrófilos en anemia megaloblástica. sobre todo en aquellos pacientes que pertenecen a los estratos socioeconómicos deprimidos.

3) Promover el uso de fármacos antirretrovirales en aquellos pacientes con infección VIH en el estadio SIDA, con el fin de disminuir su morbimortalidad.

4) Administrar suplementos multivitamínicoso de hierro en los casos de anemia carencial en los pacientes con infección VIH.

5) Realizar trabajos de investigación con poblaciones más amplias con fin de esclarecer la etiología del síndrome anémico de los pacientes peruanos en el estadio SIDA; así como describir las manifestaciones hematológicas en esta población, ya que el protocolo del Programa de atención integral al paciente infectado con VIH/SIDA aún no las contempla.

\section{AGRADECIMIENTOS}

Estudio realizado con el apoyo del Instituto de Investigaciones Clínicas-UNMSM y Laboratorio Clínico Blufstein-Lima.

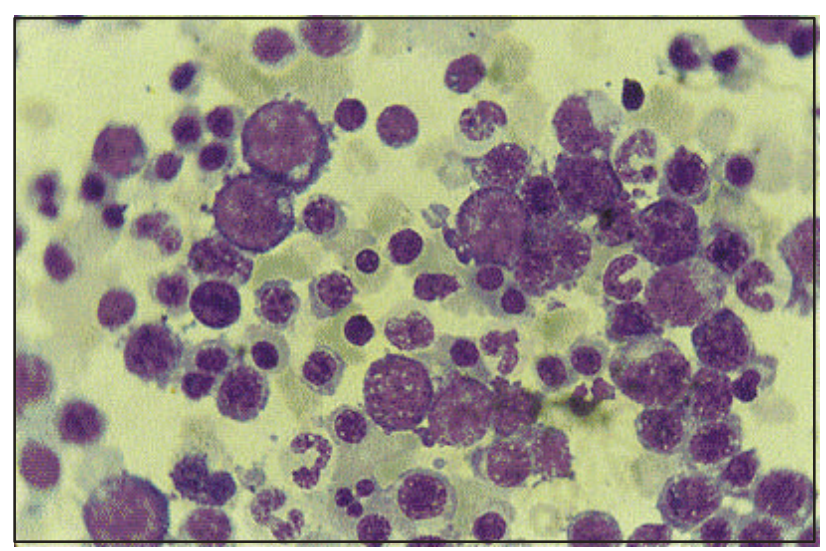

Figura 6.- Mielograma: detención en la maduración en las series eritroide y mieloide en anemia megaloblástica. 


\section{BIBLIOGRAFÍA}

1. Procetss. Guía Nacional de Atención Integral del Paciente con Infección VIH/SIDA. Ministerio de Salud - Lima; 1999.

2. Sanchez J, et al. SIDA: Epidemiología diagnóstico, tratamiento y control de la infección VIH/SIDA. Lima 1994.

3. Fannig M. HIV Infection- A Clinical Approach. WB Saunders Company; 1997.

4. Lee. Wintrobe's Clinical Hemtology. 10 Edición. Lippincort Williams \& Wilkins; 1999.

5. Aboulafia DM, Mitsuyasu RT. Hematological abnormalities in AIDS. Hematol Oncol Clin North Am 1991; 5: 195.

6. Nair J, Belleuve R, Bertoni M. Thrombotic thromboctopenic purpura in patients with the acquired immunodeficiency syndrome-related complex. Ann Intern Med 1988; 109: 209.

7. Badley AD, Pilon AA, Landay A, Lynch DH. Mechanisms of HIV-associated lymphocite apoptosis. Blood 2000: 96: 2951-64.

8. Bei-Ali Z, Dufour V, Najean Y. Platelet kinetics in human immunodefiency induced thrombocytopenia. Am J Hematol 1987; 26: 299.

9. Boyko WJ, Schecter MT, Constance P. Limited usefulness of lymphocytopenia in screening for AIDS in a hospital population. Can Med Assoc J 1985; 133: 293.

10. Shaper AG, Lewis P. Genetic neutropenia in people of African origin. Lancet 1971; 2: 1021.

11. Hochster H, Dieterich D, Bozzette S. Toxicity of combined ganciclovir and zidovudine for cytomegalovirus disease associated with AIDS. Ann Inter Med 1990; 113: 111.

12. Salama A, Lohmeyer J, Seeger W. High dose IgG for neuropenic patients with acquired immunodeficiency syndrome. Ann Hematol 1991; 63: 77.

13. Perret B, Baumgartner C. Workshop on immunotherapy of lymphoproliferative syndromes, mainly AIDS-related complex and AIDS. Von Sang 1986; 52: 1 .

14. Oyaizu N, McCloskey TW, Coronesi N. Accelerated apoptosis in peripheral blood mononuclear cells from human ummunodeficiency tyoe-1 infected patients. Blood 1993; 82: 3392.

15. Waxman S, Gang M, Goldfrank L. Tuberculosis in the HIV infected patients. Emerg Med Clin North Am 1995; 13: 1 179-98.

16. Richmann DD, Fischl MA,Grieeco MH. The toxicity of azidothymidine in the treatment of patients with AIDSS and AIDS-relaxed complex. N England J Med 1987; 317: 192.

17. Sandler AS, Kaplan LD. Diagnosis and management of systemic NHL in HIV disease. Hematol Oncol Clin N Am 1996: 5: 1111-24.

18. Vergis EN, Mellors JW. Natural history of HIV-1 infection. Infect Dis Clin N Am 2000: 4: 809-25.

19. Stone Valeri. Primary care of the patient with HIV/AIDS. Infect Dis Clin N Am 2000: 4: 967.

20. Henry DH, Beall GN, Benson CA, Carey J, Cone LA, Eron LJ, et al. Recombinant human erythropoietin in the treatment of anemia associated with human immunodeficiency virus (HIV) infection and zidovudine therapy. Overview of four clinical trials. Ann Internal Med 1992: 117: 739-48

21. Harriman G, Smith P, Horne M. Vitamin B12 malabsorption in patients with acquired immunodeficiency syndrome. Arch Intern Med 1989; 140: 2039.

22. Bennett JM, Catovsky D, Daniel MT, Flandrin G, Galton DA, Gralnick HR, et al. Proposals for the classification of the myelodysplastic syndromes. Br J Haematol 1982; 51: 189-99.

23. Thomas Coyle.Hematologic complications of HIV infection and AIDS. Med Clin N Am 1997; 2: 449.

24. Gupta S, Innan A, Licorish K. Serum ferritin in acquired inmune deficiency syndrome. J Clin Lab Immunol 1986; 20: 11 .

25. Henry DH, Beall GN, Benson CA, Carey J, Cone LA, Eron LJ, et al. Recombinant human erythropoietin in the treatment of anemia associated with human immunodeficiency virus (HIV) infection and zidovudine therapy. Ann Intern Med 1992; 117: 739-48. 